\title{
INFLUÊNCIA DE DIFERENTES MATERIAIS DE COBERTURA NO CONFORTO TÉRMICO DE INSTALAÇÕES PARA FRANGOS DE CORTE NO OESTE PAULISTA
}

\author{
JULIANO FIORELLI ${ }^{1}$, RICARDO DA FONSECA ${ }^{2}$, JOSÉ A. B. MORCELI ${ }^{3}$, \\ ANTONIO A. DIAS ${ }^{4}$
}

\begin{abstract}
RESUMO: Este trabalho apresenta um estudo da influência de diferentes materiais de cobertura no conforto térmico de instalações destinadas à criação de frangos de corte. A pesquisa foi desenvolvida no Câmpus Experimental da UNESP de Dracena - SP. Quatro protótipos em escala real foram construídos, com área de $28 \mathrm{~m}^{2}$ cada, cobertos com telha reciclada à base de embalagens longa vida, telha cerâmica, telha cerâmica pintada de branco e telha de fibrocimento. Os dados foram coletados durante o período de inverno de 2007, totalizando 90 dias. Com esses dados, foram calculados os índices de conforto térmico Carga Térmica Radiante (CTR) e a variável ambiental (Ta). Uma análise estatística por inferência e descritiva foi realizada com os valores do índice de conforto térmico e da variável ambiental. Com os resultados obtidos, é possível afirmar que a telha reciclada apresentou índices de conforto térmico semelhantes àqueles encontrados para as telhas cerâmicas. O protótipo coberto com telha de fibrocimento apresentou os maiores índices, e o coberto com telha cerâmica branca, os menores índices de conforto térmico. No entanto para o período de inverno e para os horários avaliados, todas as instalações apresentaram índices de conforto térmico fora da zona de termoneutralidade do frango de corte.
\end{abstract}

PALAVRAS-CHAVE: ambiência, frango de corte, instalações e cobertura.

\section{INFLUENCE OF ROOF MATERIAL ON THERMAL COMFORT IN BROILER HOUSINGS IN THE STATE OF SÃO PAULO}

\begin{abstract}
This paper presents a different roof tiles influence study on the thermal comfort for broiler housings. The research was conducted at UNESP's Experimental Campus at Dracena, state of São Paulo, Brazil. Four prototypes in real scale were built, each with an area of $28 \mathrm{~m}^{2}$. The prototype was covered with roof tiles made of recycled long-life packing material, ceramic tiles, ceramic tiles painted with white coating, and fiber/cement tiles. Temperatures inside the structures were recorded in the winter 2007 over a 90-day period. The results obtained indicated that recycled tile thermal behavior was similar to ceramic tiles. However for the winter period all the prototypes had presented comfort thermal index not in the broilers thermo neutral zone.
\end{abstract}

KEYWORDS: thermal comfort, broilers, housing, roof.

\section{INTRODUÇÃO}

A indústria de frangos de corte no Brasil vem apresentando forte dinamismo no que diz respeito a fatores produtivos e comerciais. Analisando-se os dados do período de 1995 a 2004 , observa-se que o País se destacou no mercado internacional desse produto em relação às taxas de crescimento mundiais. De acordo com dados do USDA $(1998,2005)$, o Brasil foi o país que apresentou maior taxa de crescimento de produção (103\%) em relação ao crescimento de $17 \%$ na produção mundial. Nas exportações, os números são ainda mais expressivos, apontando crescimento de $463 \%$, em relação à taxa de crescimento mundial, de $33 \%$. Esse crescimento nas exportações permitiu ao Brasil ocupar a posição de maior exportador mundial no ano de 2004.

\footnotetext{
${ }^{1}$ Prof. MS-3, Faculdade de Zootecnia e Engenharia de Alimentos, Universidade de São Paulo, julianofiorelli@usp.br.

${ }^{2}$ Prof. Doutor, Câmpus Experimental de Dracena, Universidade Estadual Paulista, ricardo@dracena.unesp.br.

${ }^{3}$ Zootecnista, maringa@click21.com.br.

${ }^{4}$ Prof. MS-3, Escola de Engenharia de São Carlos, Universidade de São Paulo, dia@sc.usp.br.

Recebido pelo Conselho Editorial em: 22-6-2008

Aprovado pelo Conselho Editorial em: 13-6-2010
} 
O conforto térmico no interior de instalações avícolas é fator altamente importante, pois condições climáticas inadequadas afetam consideravelmente a produção de frangos de corte. Dentro deste contexto, vários estudos já foram desenvolvidos nacional e internacionalmente, procurando avaliar diferentes materiais de cobertura e sistemas de acondicionamento térmico, com destaque para BAÊTA \& SOUZA (1997), MOURA \& NÄ̈̈S (2000), TINÔCO (2001), NÄ̈̈S et al. (2001), SILVA \& SEVEGNANI (2001), HUYNH et al. (2006) e JACOMÉ et al. (2007).

Procurando propor a utilização de materiais de construção alternativos para o meio rural, proveniente de fontes renováveis e sustentáveis, o presente trabalho avaliou a influência de diferentes materiais de cobertura, dentre os quais telha reciclada à base de embalagens longa vida, no conforto térmico de instalações zootécnicas destinadas à criação de frangos de corte.

\section{MATERIAL E MÉTODOS}

Foram edificados quatro protótipos no Câmpus da Unesp de Dracena - SP ( $21^{\circ} 20^{\prime}$ latitude sul, $51^{\circ} 52^{\prime}$ longitude oeste, $421 \mathrm{~m}$ altitude), com $8,00 \mathrm{~m}$ de comprimento e $4,00 \mathrm{~m}$ de largura, totalizando uma área coberta de $28 \mathrm{~m}^{2}$. A temperatura média durante o inverno de 2007 foi de $22{ }^{\circ} \mathrm{C}$, a velocidade média do vento $1,5 \mathrm{~m} \mathrm{~s}^{-1}$ e a radiação solar máxima média no período em estudo $560 \mathrm{~W} \mathrm{~m}^{-2}$. Os protótipos foram cobertos com telha cerâmica (TC), telha cerâmica pintada de branco com tinta à base de PVA (TCB), telha reciclada à base de embalagens longa vida (TR) e telha de fibrocimento (TFC). O sistema estrutural foi executado em eucalipto roliço, pé-direito de $3,00 \mathrm{~m}$ e cobertura com inclinação de $20 \%$. A maior dimensão foi locada no sentido leste-oeste. $\mathrm{Na}$ Figura 1, ilustram-se os protótipos avaliados.

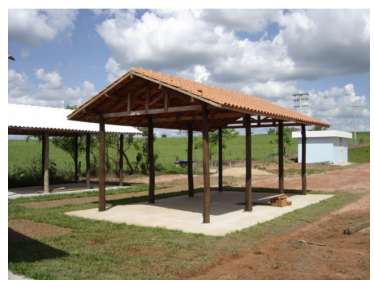

Telha cerâmica

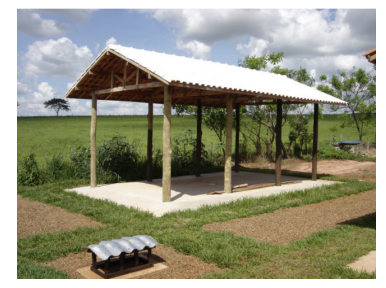

Telha cerâmica branca

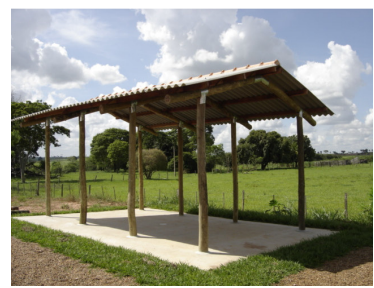

Telha de fibrocimento

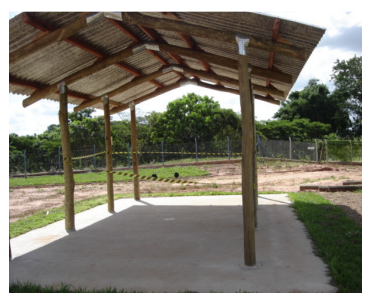

Telha reciclada

FIGURA 1. Protótipos avaliados com diferentes tipos de materiais de cobertura. Evaluated prototypes with distinct roofing types.

Para avaliar a eficiência térmica dos materiais de cobertura, foram instalados, em cada um dos protótipos, um termômetro de máxima e mínima (1,5 $\mathrm{m}$ do piso), dois termômetros de bulbo seco e bulbo úmido ( $0,7 \mathrm{~m}$ e 1,5 $\mathrm{m}$ do piso), um anemômetro digital portátil e dois termômetros de Globo Negro (GN) (0,7 m e 1,5 m do piso). Para caracterizar o ambiente do experimento, foi utilizada uma estação meteorológica da marca DAVIS ${ }^{\circ}$, Vantage PRO2. As coletas foram realizadas nos horários das 10h, 12h, 14h e 16h, durante o inverno de 2007, totalizando 90 dias. Foi escolhido o período de inverno, pois, segundo SANDRE et al. (2008), esta é a época do ano em que a região onde o experimento foi realizado apresenta condições climáticas mais amenas, com temperaturas médias em torno de $22{ }^{\circ} \mathrm{C}$ (temperatura média mínima de $16{ }^{\circ} \mathrm{C}$ e máxima de $26{ }^{\circ} \mathrm{C}$ ), consequentemente, período do ano em que o animal tende a apresentar menor estresse térmico.

Foram determinados os índices de conforto térmico Carga Térmica Radiante (CTR), por meio da equação de ESMAY (1969) e Temperatura Ambiente (Ta).

Com os dados obtidos, foi realizada uma análise estatística, segundo o delineamento em blocos casualizados (DBC), no esquema fatorial 4x4, em que os fatores estudados corresponderam ao acondicionamento térmico e horas de coletas. $\mathrm{O}$ fator acondicionamento térmico constituiu-se de quatro níveis (TR, TFC, TC e TCB), e o fator horas de coletas compreendeu quatro níveis (10h, $12 \mathrm{~h}, 14 \mathrm{~h}$ e $16 \mathrm{~h}$ ), totalizando dezesseis tratamentos. As repetições de cada tratamento foram consideradas os dias de coletas dos dados climáticos. 
A eficiência dos protótipos foi fundamentada na avaliação dos índices de conforto térmico CTR e na variável climática Temperatura Ambiente (Ta), por se tratar de construções abertas, onde possíveis diferenças na umidade do ar entre as construções não têm significado real, pois a ventilação é livre, e a interação com a atmosfera externa é total. Para melhor compreensão do modelo estatístico empregado na análise dos resultados, a Tabela 1 apresenta os níveis dos fatores estudados (acondicionamento térmico e horas de coletas) e os respectivos tratamentos. Cada quadrado colorido da Tabela 1 equivale a um tratamento estudado.

TABELA 1. Modelo estatístico adotado no experimento. Statistic model adopted for the experiment.

\begin{tabular}{ccccccc}
\hline & \multicolumn{5}{c}{ Níveis do Fator Horas } \\
\hline \multirow{3}{*}{ Níveis do fator } & TR & TR-10 & TR-12 & TR-14 & TR-16 \\
\cline { 2 - 6 } acondionamento & TFC & TFC-10 & TFC-12 & TFC-14 & TFC-16 \\
& TC & TC-10 & TC-12 & TC-14 & TC-16 \\
& TCB & TCB-10 & TCB-12 & TCB-14 & TCB-16 \\
\hline
\end{tabular}

O índice de conforto térmico (CTR) e a variável ambiental (Ta) foram analisados pela eq.(1):

$$
\mathrm{Y}_{\mathrm{ijkl}}=\mu+\mathrm{t}_{\mathrm{i}}+\mathrm{d}_{\mathrm{j}}+\mathrm{h}_{\mathrm{jk}}+(\mathrm{td})_{\mathrm{ij}}+\varepsilon_{\mathrm{ijkl}}
$$

em que,

$\mathrm{Y}_{\mathrm{ijk}}$ - k-ésima observação da variável CTR e Ta, no j-ésimo nível do fator horário e no i-ésimo nível do fator acondicionamento;

$\mu$ - média geral;

ti - efeito do tipo de cobertura $(\mathrm{i}=1, \ldots, 4)$;

$\mathrm{dj}$ - efeito de dia $(\mathrm{j}=1, \ldots, 90)$;

hjk - efeito de hora dentro de dia $(\mathrm{k}=1, \ldots, 4)$;

$(\mathrm{td})_{\mathrm{ij}}$ - efeito da interação cobertura x horário, e

عijk - efeito residual.

Os dados qualitativos (fatores tratamentos e horas) foram analisados por meio do teste de comparação múltipla (Tukey), quando a ANOVA foi significativa, sendo ambos testados a $\mathrm{P}<0,05$. Os resultados obtidos foram avaliados e interpretados pelo sistema para análises estatísticas "Restatística".

\section{RESULTADOS E DISCUSSÃO}

\section{Análise das coberturas}

Na Figura 2, apresenta-se análise estatística descritiva que contempla a observação da CTR e $\mathrm{T}_{\mathrm{a}}$ referente às diferentes telhas em estudo, em função dos horários avaliados, para a altura do termômetro de Globo Negro 0,30 m e 0,70 m do piso.

Pela análise da variância (ANOVA), observou-se que a interação cobertura:horas não foi significativa para CTR 1 e CTR 2. Sendo assim, a análise foi realizada isolada e foram identificados valores de $\mathrm{F}$ significativos $(\mathrm{p}<0,05)$ para as variáveis dia, tratamento e horas. 


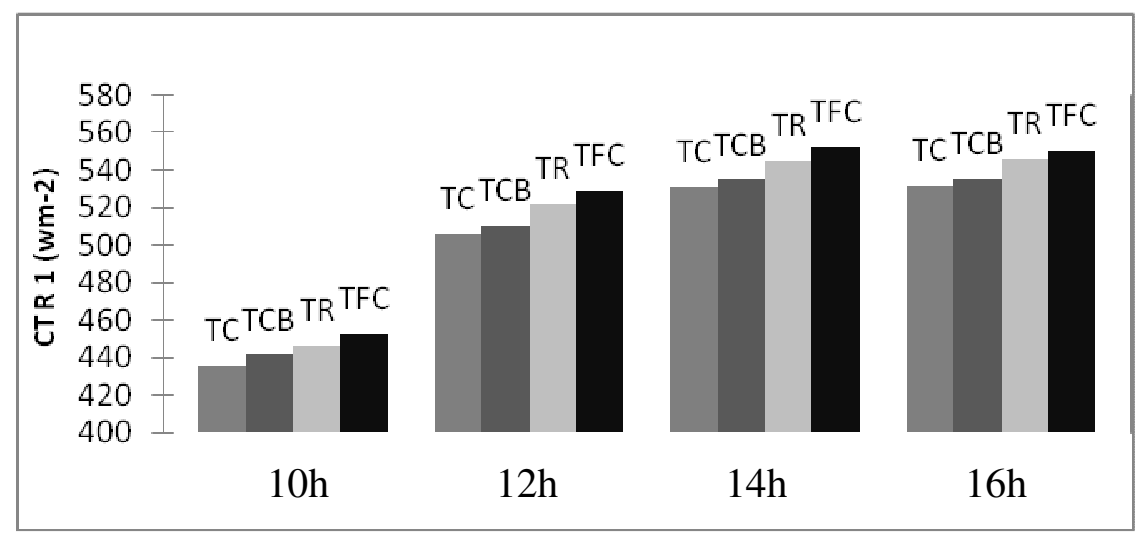

A. Valores médios da CTR 1 (0,30 $\mathrm{m}$ do solo) versus horário de leitura

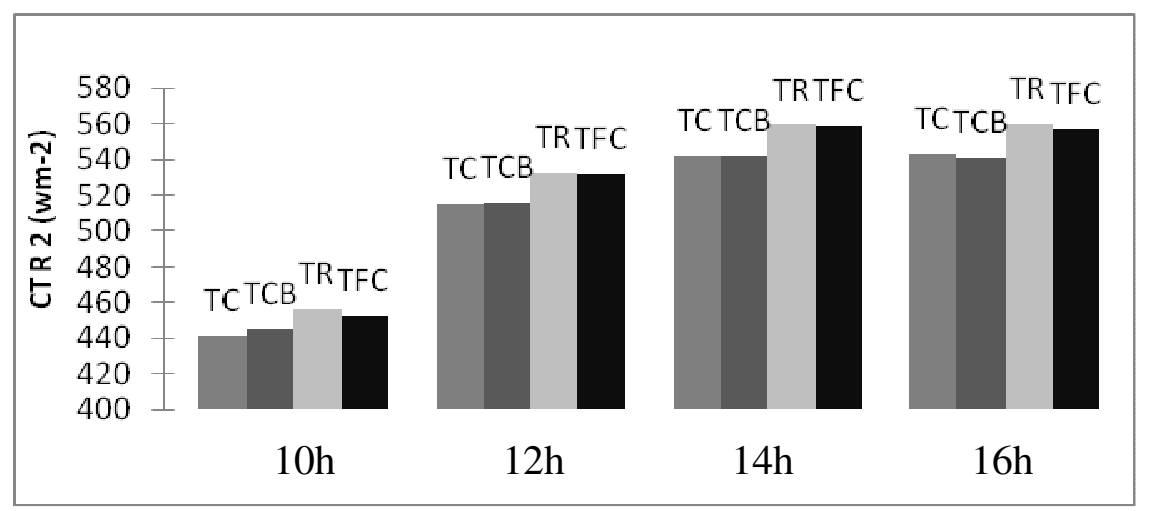

B. Valores médios da CTR 2 (0,70 $\mathrm{m}$ do solo) versus horário de leitura

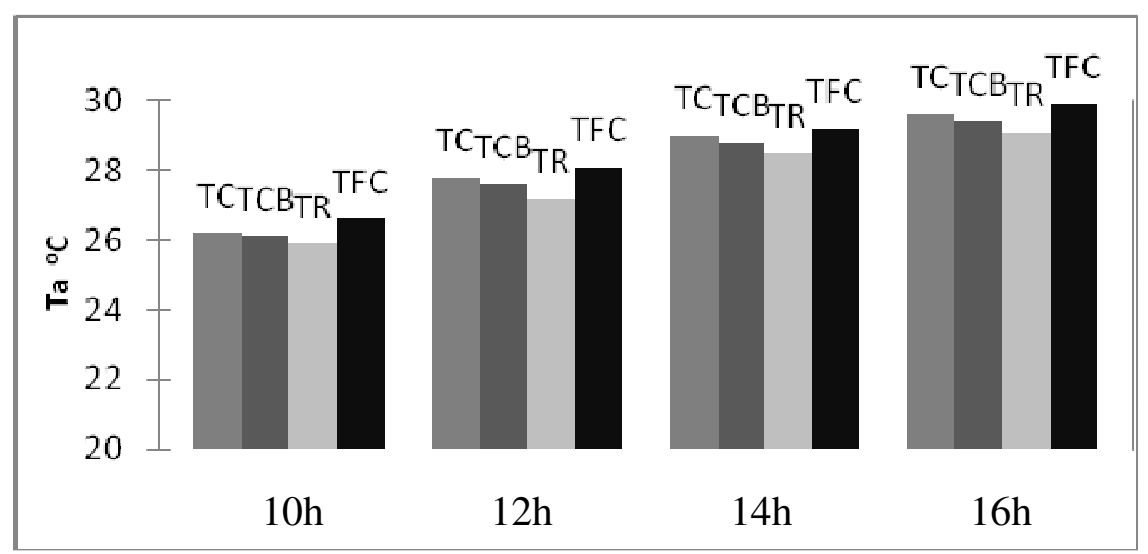

C. Valores médios de $\mathrm{T}_{\mathrm{a}}$ versus horário de leitura

FIGURA 2. Valores médios de carga térmica radiante e temperatura ambiente. Average values of thermal radiation load and environment temperature.

Com base na análise da ANOVA, procedeu-se ao teste de Tukey, a 5\% de probabilidade, para as variações (dia, tratamento e horas), com o objetivo de investigar o comportamento das médias.

Na Tabela 2, são apresentados os valores médios e desvio-padrão de CTR e $\mathrm{T}_{\mathrm{a}}$ para cada tipo de telha avaliada e para os diferentes horários. Para a variável horas, apenas na interação 14h e 16h, não foi identificada diferença significativa para CTR 1 e CTR 2. 
TABELA 2. Valores médios de carga térmica radiante e temperatura ambiente. Average values of thermal radiation load and environment temperature.

\begin{tabular}{|c|c|c|c|c|c|c|c|c|}
\hline & \multicolumn{4}{|c|}{$10 \mathrm{~h}$} & \multicolumn{4}{|c|}{$12 \mathrm{~h}$} \\
\hline & TC & TCB & TR & TFC & $\mathrm{TC}$ & TCB & TR & TFC \\
\hline CTR $1 \mathrm{Wm}^{-2}$ & $436,16^{\mathrm{a}}$ & $441,58^{a}$ & $446,25^{\mathrm{b}}$ & $452,80^{c}$ & $506,02^{\mathrm{a}}$ & $510,25^{a}$ & $522,20^{\mathrm{b}}$ & $529,06^{c}$ \\
\hline (Desvio-Pad.) & 51,73 & 53,81 & 62,24 & 32,81 & 29,76 & 30,07 & 27,95 & 34,76 \\
\hline CTR $2 \mathrm{Wm}^{-2}$ & $441,03^{a}$ & $444,85^{\mathrm{a}}$ & $456,22^{b}$ & $452,67^{c}$ & $515,10^{\text {a }}$ & $516,06^{\mathrm{a}}$ & $532,91^{b}$ & $532,02^{c}$ \\
\hline (Desvio-Pad.) & 52,66 & 54,84 & 65,12 & 33,22 & 35,07 & 30,08 & 30,73 & 34,62 \\
\hline $\mathrm{Ta}^{\circ} \mathrm{C}$ & 26,2 & 26,1 & 25,9 & 26,6 & 27,8 & 27,6 & 27,2 & 28,1 \\
\hline \multirow[t]{3}{*}{ (Desvio-Pad.) } & 4,74 & 4,71 & 5,12 & 4,76 & 4,73 & 4,58 & 4,61 & 4,33 \\
\hline & \multicolumn{4}{|c|}{$14 \mathrm{~h}$} & \multicolumn{4}{|c|}{$16 \mathrm{~h}$} \\
\hline & $\mathrm{TC}$ & TCB & TR & TFC & $\mathrm{TC}$ & TCB & TR & TFC \\
\hline CTR $1 \mathrm{Wm}^{-2}$ & $530,61^{\mathrm{a}}$ & $534,65^{\mathrm{a}}$ & $544,88^{b}$ & $552,14^{c}$ & $531,34^{\mathrm{a}}$ & $535,40^{\mathrm{a}}$ & $545,99^{b}$ & $550,14^{\mathrm{c}}$ \\
\hline (Desvio-Pad.) & 31,75 & 34,79 & 33,16 & 35,92 & 46,93 & 38,70 & 36,93 & 39,32 \\
\hline CTR $2 \mathrm{Wm}^{-2}$ & $542,29^{a}$ & $541,63^{\mathrm{a}}$ & $559,29^{b}$ & $558,72^{\mathrm{c}}$ & $542,69^{a}$ & $541,37^{\mathrm{a}}$ & $560,04^{b}$ & $556,63^{\mathrm{c}}$ \\
\hline (Desvio- Pad) & 31,58 & 36,07 & 33,89 & 37,11 & 46,56 & 37,60 & 37,42 & 39,15 \\
\hline $\mathrm{Ta}^{\circ} \mathrm{C}$ & 29 & 28,8 & 28,5 & 29,2 & 29,6 & 29,4 & 29,1 & 29,9 \\
\hline (Desvio-Pad.) & 4,55 & 4,42 & 4,46 & 4,33 & 4,55 & 4,48 & 4,35 & 4,34 \\
\hline
\end{tabular}

Obs.: CTR 1 - medição a $0,30 \mathrm{~m}$ do solo e CTR 2 - medição a $0,70 \mathrm{~m}$ do solo. Letras diferentes indicam diferença significativa $(\mathrm{p}<0,05)$

Na Figura 2 (A e B), mostra-se o aumento no valor da CTR ao longo do dia, para todas as coberturas avaliadas, atingindo valor máximo entre $15 \mathrm{~h}$ e $16 \mathrm{~h}$.

Os menores valores de CTR ocorreram às $10 \mathrm{~h}$, e os maiores, às $12 \mathrm{~h}$ e $14 \mathrm{~h}$, respectivamente. Este comportamento diurno da CTR foi verificado por ROSA (1984), FERREIRA (1996), FONSECA (1998), ZANOLLA et al. (1999), SARTOR et al. (2000), NÄ̈̈S et al. (2001), MATOS (2001) e FURTADO et al. (2003), e é explicado pelo fato de que os valores que definem a CTR ocorrem em função da radiação solar que, por sua vez, atinge os valores mais elevados próximos de $12 \mathrm{~h}$, quando o sol se posiciona de forma mais perpendicular ao plano do horizonte local e, também, em função da radiação de ondas longas emitidas pela vizinhança (FONSECA, 1998). ABREU et al. (2009) avaliaram a eficiência de forro de polietileno em instalações avícolas confeccionadas com cobertura de fibrocimento, e os maiores valores para a CTR foram identificados nos horários próximos das $14 \mathrm{~h}$, corroborando os valores observados nesta pesquisa.

Os menores valores de CTR foram identificados no protótipo coberto com TC e TCB, e não foi identificada diferença estatística significativa entre os valores. Já os maiores valores de CTR foram identificados no protótipo coberto com TFC. O mesmo comportamento ocorreu para a temperatura ambiente, no entanto os protótipos cobertos com TR apresentaram a menor temperatura ambiente para todos os horários avaliados.

Considerando-se como referência os valores indicados por ROSA (1984) para abrigos em que se utilizaram telhas de cimento-amianto $\left(\mathrm{CTR}=515,4 \mathrm{~W} \mathrm{~m}^{-2}\right.$ ), vê-se que, para os horários mais críticos, após as 12h, CTR 1 e CTR 2 foram superiores para todas as coberturas. Os valores obtidos nesta pesquisa estão coerentes com os apresentados por FURTADO et al. (2003), que avaliaram galpões destinados à criação de frangos de corte cobertos com telha cerâmica e telha de fibrocimento-amianto. Para os horários de maior radiação, a CTR foi superior a $489,9 \mathrm{Wm}^{-2}$.

A análise estatística indicou diferença significativa entre os valores de CTR 1 e CTR 2, conforme apresentado na Tabela 2. A TR diferiu estatisticamente da TFC, e como a CTR e $\mathrm{T}_{\mathrm{a}}$ encontradas para este material foram menores, a TR apresenta-se como opção de cobertura para instalações avícolas.

Comparando os valores obtidos para variável climática $\mathrm{T}_{\mathrm{a}}$, com aqueles de termoneutralidade de frangos de corte $\left(1\right.$ dia $35{ }^{\circ} \mathrm{C}, 28$ dias $21-24{ }^{\circ} \mathrm{C}$ e 42 dias $20{ }^{\circ} \mathrm{C}$ ), é possível afirmar que o 
ambiente resultante coberto com as telhas estudadas não promove conforto térmico para as aves alojadas, em diferentes idades, em instalações da região oeste paulista, durante o período de inverno. Dentro deste contexto, recomenda-se seguir os procedimentos indicados por MOURA (1998), YAHAV (2004) e EICHEN (2008). De maneira geral, os autores afirmam que, para um bom conforto térmico no interior das instalações avícolas, é necessário utilizar sistemas de climatização. O sistema de ventilação tipo túnel por pressão positiva, utilizando linhas de nebulização longitudinalmente nos galpões, é o que vem apresentando melhores resultados em termos de climatização de aviários. Linhas de ventiladores dispostas por todo o comprimento do galpão auxiliam na movimentação do ar, que é retirado do prédio por exaustores instalados na outra extremidade do galpão.

\section{CONCLUSÕES}

Os índices de conforto térmico CTR referente às coberturas de TC, TCB, TR e TFC, para os horários avaliados variaram significativamente, a $5 \%$ de probabilidade.

A TR, classificada como um material sustentável, fabricado a partir de resíduos de caixas tetra pack, é uma nova opção de cobertura para ser utilizada em instalações avícolas, uma vez que apresentou índices de conforto térmico CTR menores e estatisticamente diferentes dos identificados para TFC.

Para o período de inverno em estudo, as quatro telhas avaliadas não apresentaram temperaturas próximas àquelas de termoneutralidade para frangos de corte, sendo necessária a adoção de sistemas adicionais de resfriamento, para diferentes horários, mesmo em períodos de inverno, na região oeste paulista.

\section{AGRADECIMENTOS}

À FAPESP e ao CNPq, pelo apoio à pesquisa.

\section{REFERÊNCIAS}

ABREU, P.G.; ABREU, V.M.N.; COLDEBELLA, A.; JAENISH, F.R.F.; PAIVA, D.P. Condições térmicas ambientais e desempenho de aves criadas em aviários com e sem o uso de forro. Arquivo Brasileiro de Medicina Veterinária e Zootecnia, Belo Horizonte, v.59, n.4, p.1.014-1.020, 2009.

BAÊTA, F.C.; SOUZA, C.F. Ambiência em edificações rurais - conforto animal. Viçosa: UFV, 1997. $246 \mathrm{p}$.

EICHEN, P.A.; LUCY, M.C.; SAFRANSKI, T.J.; COATE, E.A.; WILLIAMS, A.M.; SPIERS, D.E. Heat stress effects on sow reproductive performance using simulated forced air and evaporative cooling systems. Transaction of the ASAE, St. Joseph, p.773-779, 2008.

ESMAY, M.L. Principles of animal environment. Westport: AVI Publishing, 1969. 325 p.

FERREIRA, P.V. Estatística experimental aplicada à agronomia. 2.ed. Maceió: Editora Universitária, 1996. 604 p.

FONSECA, J.M. Efeito da densidade de alojamento sobre o desempenho de frangos de corte criados em sistemas de nebulização e ventilação em túnel. 1998. 57 f. Dissertação (Mestrado) Universidade Federal de Viçosa, Viçosa - MG, 1998.

FURTADO, D.A.; AZEVEDO, P.V.; TINÔCO, I.F.F. Análise do conforto térmico em galpões avícolas com diferentes sistemas de acondicionamento. Revista Brasileira de Engenharia Agrícola e Ambiental, Campina Grande, v.7, n.3, p.559-564, 2003.

HUYNH, T.T.T.; AARNINK, A.J.A.; VERSTEGEN, M.W.A.; GERRISTS, W.J.J.; HEETKAMPS, M.J.W.; KEMPS, B.; CANH, T.T. Effects of increasing temperatures on physiological changes in pigs at different relative humidities. Journal of Animal Sciense, Savoy, v.83, p.1.385-1.396, 2005. 
JÁCOME, I.M.T.D.; FURTADO, D.A.; LEAL, A.F.; SILVA, J.H.V.; MOURA, J.F.P. Avaliação de índices de conforto térmico de instalações para poedeiras no Nordeste do Brasil. Revista Brasileira de Engenharia Agrícola e Ambiental, Campina Grande, v.11, n.5, p.527-531, 2007.

MATOS, M. L. Conforto térmico ambiente e desempenho de frangos de corte, alojados em dois níveis de alta densidade, em galpões com sistemas de ventilação em túnel e ventilação lateral. 2001. 89 f. Dissertação (Mestrado) - Universidade Federal de Viçosa, Viçosa, 2001.

MOURA, D.J. Avaliação da eficiência térmica de instalações avícolas sombreadas e ventiladas artificialmente, em diferentes orientações. 1998. Tese (Doutorado) - Faculdade de Engenharia Agrícola, Universidade Estadual de Campinas, Campinas, 1998.

MOURA, D.J. ; NÄÄS, I.A. Avaliação da eficiência térmica de instalações avícolas sombreadas e ventiladas artificialmente, em diferentes orientações no período de verão. Brazilian Journal of Poultry Science, Campinas, v.1, n.3, p.167-174, 2000.

NÄÄS, I.A.; SEVEGNANI, K.B.; MARCHETO, F.G.; ESPELHO, J.C.C.; MENEGASSI, V.; SILVA, I.J.O. Avaliação térmica de telhas de composição de celulose e betume, pintadas de branco, em modelos de aviários com escala reduzida. Engenharia Agrícola, Jaboticabal, v.21, n.2, p.121126, 2001.

ROSA, Y.B.C.J. Influência de três materiais de cobertura no índice de conforto térmico em condições de verão, para Viçosa. 1984. 77 f. Dissertação (Mestrado) - Universidade Federal de Viçosa, Viçosa - MG, 1984.

SANDRE, L.C.G.; FIORELLI, J.; FONSECA, R.; PELOSO, L.A.; RIZZI, V. Diagnóstico de temperatura e precipitação no município de Dracena - SP, nas últimas décadas. In. SEMINÁRIO DE CONSTRUÇÕES RURAIS E AMBIÊNCIA APLICADAS À PRODUÇÃO ANIMAL, 1., 2008, Tupã. Anais... 1 CD-ROM.

SARTOR, V.; BAETA, F.C.; ORLANDO, R.C.; LUZ, M.L.; TINÔCO, I.F.F. Efeito de sistemas de resfriamento evaporativo em instalações para frangos de corte. Engenharia Agrícola, Jaboticabal, v.20, n.2, p.87-97, 2000.

SILVA, I.J.O.; SEVEGNANI, K.B. Ambiência na produção de aves de postura. In: SILVA, I.J.O. Ambiência na produção de aves em clima tropical. Jaboticabal: SBEA, 2001. p.150-214.

TINÔCO, I. F. F. Avicultura industrial: novos conceitos de materiais, concepções e técnicas construtivas disponíveis para galpões avícolas brasileiros. Revista Brasileira de Ciência Avícola, Campinas, v.3, n.1, p.1-26, 2001.

USDA. UNITED STATES DEPARTMENT OF AGRICULTURE. World Markets and Trade. Total poultry meat production. 1998. Disponível em: <http://www.usda.gov>. Acesso em: 13 maio 2005.

USDA. UNITED STATES DEPARTMENT OF AGRICULTURE. Broiler summary selected countries. 2005. Disponível em: <http://www.usda.gov>. Acesso em: 13 maio 2005.

YAHAV, S.; STRASCHNOW, A.; LUGER, D.; SHINDER, D.; TANNY, J.; COHEN, S. Ventilation, sensible heat loss, broiler energy, and water balance under harsh environmental conditions. Poultry Science, Savoy, v.83, p.253-258, 2004.

ZANOLLA, N.; TINÔCO, I.F.F.; BAÊTA, F.C.; CECON, P.R.; MORAES, S.R.P. Sistemas de ventilação em túnel e lateral na criação de frangos de corte em alta densidade. Revista Brasileira de Engenharia Agrícola e Ambiental, Campina Grande, v.3, n.3, p.361-366, 1999. 